Rev. High Pressure Sci. Technol., Vol. 7 (1998) 1511 1513

\title{
Non-hydrostatic Conditions in High-pressure Devices: Analysis of Plastic Deformation with EDX
}

\author{
J.W.Otto I, J.K.Vassiliou and G.Frommeyer ${ }^{*}$ \\ I Dept. Physics, Villanova University, Villanova, PA 19085, USA \\ ${ }^{*}$ MPI Eisenforschung, 40237 Düsseldorf, Germany
}

\begin{abstract}
The effect of different pressure devices and pressure media on the compression behavior of elastically anisotropic polycrystals $\left(\mathrm{Cu}_{3} \mathrm{Au}, \mathrm{Ni}_{3} \mathrm{Al}\right)$ is investigated with energy-dispersive X-ray diffraction (EDX) correlating information from both line profiles and line positions. Plastic deformation due to stacking faults leads to a volume incompressibility preceded at high ratios of non-hydrostatic to hydrostatic stresses by a volume increase due to introduction of dislocations. A discontinuity in the compressibility at the transition back from plastic to elastic compression is due to the yield strength of the plastically deformed material and corresponds to the Hugoniot elastic limit. The compression of a fully plastically deformed material is elastic and isotropic, independent of the presence and type of pressure medium. The yield strength of the samples is estimated from the offset to the hydrostat of the compression curve above the discontinuity in the compressibility.

[diamond anvil cell, energy-dispersive X-ray diffraction, plastic deformation, pressure calibration]
\end{abstract}

\section{Introduction}

Deviations from hydrostatic conditions in high-pressure experiments can occur due to a non-isotropic macroscopic stress field set-up by the pressure device, substantial viscosity of the pressure transmitting medium or local stresses set up at the grain boundaries of a polycrystalline elastically anisotropic sample. The viscosity at $5 \mathrm{GPa}$ for 1:1 pentane-isopentane and at $7-8 \mathrm{GPa}$ for methanol is of the order of $10^{-2} \mathrm{GPa} \mathrm{s}$, an increase of $10^{8}$ over the value at ambient conditions [1]. The critical resolved shear stress for the glide of dislocations in single crystals (and hence for plastic deformation by slip) is of the order of only $10^{-4}-10^{-3} \mathrm{GPa}$ for ductile fcc-based metals at ambient conditions, for example. The yield stress in polycrystals is higher than this value by a factor of 3 for fcc and bcc based materials and may increase substantially with decreasing grain size (Hall-Petch relation) [2]. The pressure effect on the yield stress is controversial [3]. Nevertheless, stresses of only a.small fraction of the applied nominally hydrostatic pressure are required to cause plastic deformation of many materials and such stresses may occur even in some liquid pressure media. The purpose of our work is to investigate the origins of non-hydrostatic stresses in high-pressure experiments and their consequences correlating information from both line positions and line profiles.

\section{Experimental}

Polycrystalline foils of disordered $\mathrm{Cu}_{3} \mathrm{Au}$ and ordered $\mathrm{Ni}_{3} \mathrm{Al}$ 30-50 $\mu \mathrm{m}$ thick were compressed in a diamond anvil cell (DAC) both without and with a pressure medium $\left(\mathrm{Cu}_{3} \mathrm{Au}\right.$ with $\mathrm{NaCl}$, paraffin oil and 4:1 methanol-ethanol; $\mathrm{Ni}_{3} \mathrm{Al}$ with $\mathrm{NaCl}$ ), and in a multi-anvil apparatus (MAX80; $\mathrm{Ni}_{3} \mathrm{Al}$ with $\mathrm{NaCl}$ ). The crystallite sizes in the foils produced by splat-quenching were estimated from line broadening to be in the range $0.2-5 \mu \mathrm{m}$, providing for good powder averaging.
$\mathrm{Ni}_{3} \mathrm{Al}$ and $\mathrm{Cu}_{3} \mathrm{Au}$ crystallize in an fcc superlattice structure $\left(L 1_{2}\right)$. The yield strengths of polcrystals of $1 \mu \mathrm{m}$ in size are $0.47 \mathrm{GPa}$ and $2.2 \mathrm{GPa}$ for disordered $\mathrm{Cu}_{3} \mathrm{Au}$ and for plastically deformed ordered $\mathrm{Ni}_{3} \mathrm{Al}$, respectively. The tensile yield stress for single crystals of disordered $\mathrm{Cu}_{3} \mathrm{Au}$ is 0.02 $\mathrm{GPa}$ and the $0.2 \%$ flow stress for ordered polycrystalline $\mathrm{Ni}_{3} \mathrm{Al}$ is $0.13 \mathrm{GPa}$ [4]. Deformation results in introduction of stacking and twin faults on $\{111\}$ planes and (in the ordered state) of anti-phase boundaries on $\{001\}$ planes [4]. Experiments were carried out at beamlines F3 (DAC) and F2 (MAX80) at the Hamburg Synchrotron Radiation Laboratory using energy-dispersive techniques. Pressures were measured by the ruby fluorescence technique (DAC) and from $\mathrm{NaCl}$ using the Decker equation of state (DAC, MAX80).

3. Analyzing lattice deformations with EDX

In the elastic regime, the uniaxial stress component can be calculated from the measured shifts in lattice spacings $d_{h k l}-d_{o}(h k l) / d_{o}(h k l)$ of a cubic substance as a function of direction in the lattice (expressed by the factor $\Gamma$ ) [5]. Multiple orders of a reflection (having the same $\Gamma$ ) should be shifted by the same amount for elastic strains, and by unequal amounts with alternating sign by introduction of stacking faults on $\{111\}$ planes during plastic deformation [6]. When multiple orders of a reflection are not available or are not reliable, the stacking fault probability can be determined from the change in separation of two reflections (such as $\Delta E(200-111)$ ) [6]. Since the shift due to stacking faults is largest for the (200) and (111) reflections and since the $\Gamma$ of these reflections are the extreme values in the fcc lattice, the stacking faults may determine the uniaxial stress component as calculated with the Kennedy-Singh approach. A probability of stacking faults of $1 / 51$ in $\mathrm{Cu}_{3} \mathrm{Au}$ results in a residual stress of $0.55 \mathrm{GPa}$ calculated with 
the Kennedy-Singh equation (compare Figs. $1 \mathrm{~b}$ and 1c). Line profiles in principle contain information about size and strain [6]. When multiple orders of reflections are not available or reliable for a Williamson-Hall analysis, an effective crystallite size is often estimated from the Lorentzian component and a strain $\epsilon$ from the Gaussian component of a single peak profile using the Scherrer and Stokes-Wilson equations, respectively. A proper calculation for elastically anisotropic solids does require multiple orders of reflections. The effective crystallite size contains contributions from stacking faults, twin faults and (in partially ordered materials) anti-phase boundaries [6]. Twin faults in addition leak to peak asymmetries but these may be masked in EDX by electronic effects in the detector and counting circuitry. The peak profile in EDX with synchrotron radiation and tight collimation of incident and diffracted beams is essentially a pure Gaussian [7]. Plastic deformation in $\mathrm{Cu}_{3} \mathrm{Au}$ and $\mathrm{Au}$ was shown to lead to strong line broadening and a dominant Lorentzian character of the peak profiles [8]. Voigt functions are therefore being used in our studies. The resolution of EDX with synchrotron radiation requires a stacking fault probability of $\geq 1 / 200$, an effective crystallite size $\leq 0.2-0.5 \mu \mathrm{m}$ and strains $\geq 10^{-3}$ for detection.

\section{Results}

The compression behavior was found to depend on the ratio of shear to hydrostatic stress. The initial compression is elastic (Figs. 1-3) but hydrostatic only when a pressure medium is being used (Figs. 2,3). High ratios of uniaxial to hydrostatic stress during direct compression of a foil of $\mathrm{Cu}_{3} \mathrm{Au}$ between diamonds lead to a volume increase which can be traced to the introduction of dislocations [9]. Deviations in the lattice parameters calculated from individual lattice spacings (Fig. 1a-c), increasing linewidths and increasing Lorentzian character of the line profiles (Fig. 1d) and the irreversibility of these changes on pressure release indicate plastic deformation by the introduction of stacking faults (Fig. 1c). These may arise by the splitting of dislocations, for example. A region of incompressibility (Fig. 1 between 0.8 and $1.3 \mathrm{GPa}$; Figs. 2,3) is interpreted to be due to work hardening after the yield strength of the previously undeformed solid has been exceeded. Changes in line profiles and deviations in lattice parameters saturate around a discontinuity in the compressibility (Fig. 1). The discontinuity is interpreted as being due to the yield strength of the fully plastically deformed solid. Above the discontinuity, the compression is elastic and isotropic (Fig. 1). The effective crystallite size calculated from the Lorentzian integral widths varies from 45 to $15 \mathrm{~nm}$ and the strains calculated from the Gaussian integral widths vary from 0.003 to 0.005 for the (111) and (311) reflections, respectively, at $6.7 \mathrm{GPa}$. On pressure release, the lattice spacings trace a path also followed on compression of the plastically deformed solid (Fig. 2)[10]. Note that the average of the compression-decompression cycle

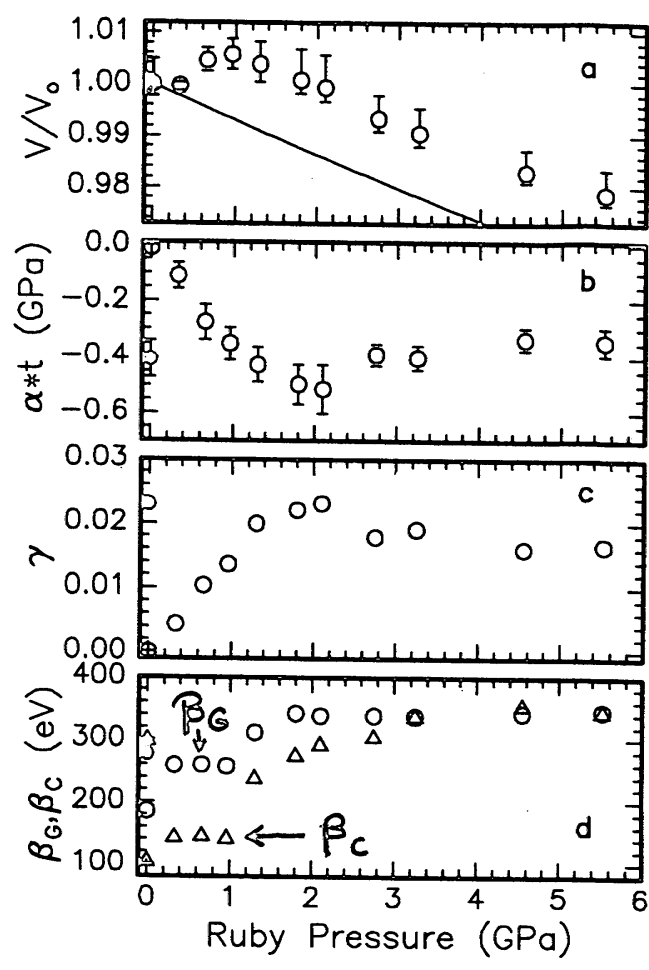

Fig. 1. Direct compression of disordered $\mathrm{Cu}_{3} \mathrm{Au}$ foil between diamonds. Open and solid symbols for increasing pressure and after pressure release, respectively. Other decompression results are not shown for reasons of clarity. From top to bottom, average volume $\left(\mathrm{V} / \mathrm{V}_{0}\right.$ of $(111)$ and (200) represent the lowest and highest values observed and are indicated by the lower and upper error bar, respectively), uniaxial stress component $\alpha$ t (where $\alpha$ is the fraction of the Reuss state), stacking fault probability $\gamma$ calculated from the separation of the (111) and (200) spacings, and the Gaussian $\left(\beta_{G}\right)$ and Lorentzian $\left(\beta_{C}\right)$ integral widths for the $(200)$ reflection corrected for the instrumental profile.

does not conform to the equation of state (Figs. 2,3). The initial deviation from the equation of state on compression (Fig. 3), the beginning of work hardening (Figs. 2,3 ) and the discontinuity in the compressibility are shifted up in pressure when the ratio of shear to hydrostatic stress is decreased by the use of a weaker solid (Fig. 2) or of weaker pressure transmitting media (Fig. 3) (compare also Le Bihan etal.[11]). The compression behavior is the same for $\mathrm{Ni}_{3} \mathrm{Al}$ in $\mathrm{NaCl}$ in a $\mathrm{DAC}$ and in a multi-anvil press (Fig. 2, bottom). The loci of the discontinuities in the compression curves and of the volume data above the discontinuity define a single curve (Fig. 3) indicating a compressibility similar to that of the undeformed solid under hydrostatic conditions. The (constant) offset to the 


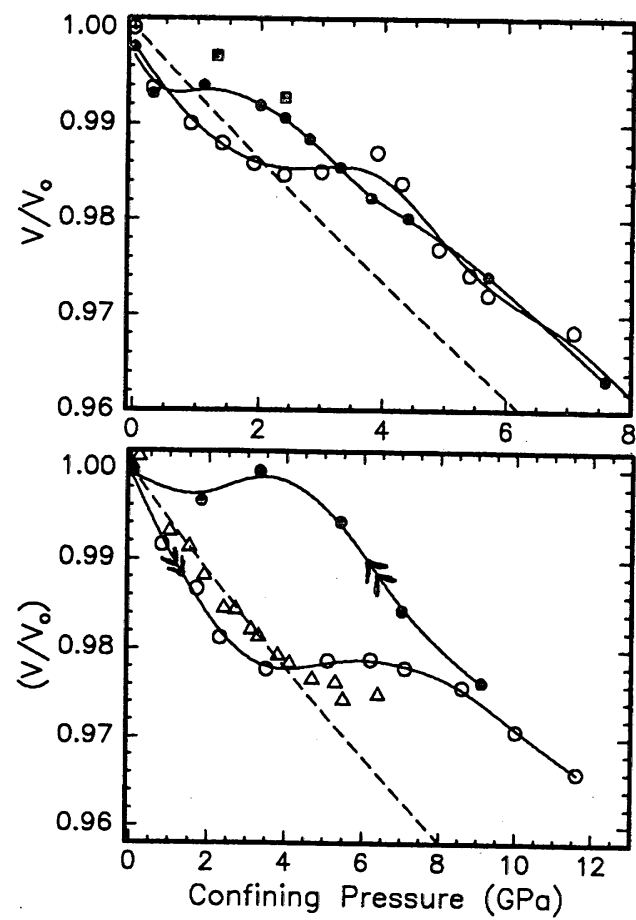

Fig. 2. Average scaled volume for disordered $\mathrm{Cu}_{3} \mathrm{Au}$ (top)[10] and ordered $\mathrm{Ni}_{3} \mathrm{Al}$ (bottom) in $\mathrm{NaCl}$ in the DAC (circles) and the MAX80 (triangles). Open and solid circles are for pressure increase and decrease, respectively, and solid squares for recompression. The solid lines are spline fits to the volume data, the dashed curves are the ultrasonically determined equations of state.

hydrostat is around $1.9 \mathrm{GPa}$ for $\mathrm{Cu}_{3} \mathrm{Au}$ (Figs. 2 top, 3 ) and is estimated to be $4-5 \mathrm{GPa}$ for $\mathrm{Ni}_{3} \mathrm{Al}$ (Fig. 2, bottom). A discontinuity in the compressibility has also been observed in shock-wave studies of polycrystalline materials (the Hugoniot elastic limit) [12]. For an elastically isotropic material, the difference in pressure at which the same volume would be observed under hydrostatic and pure uniaxial stress is $2 / 3 \mathrm{Y}$ if the von Mises yield criterion is used [12]; if the compression curve above the yield point contains a hydrostatic component, the offset is $(\mathrm{K} / 2 \mu) \mathrm{t}$. The value of $Y$ calculated in either way is far above the yield strength calculated from $\alpha$ t with the von Mises criterion (Fig. 1b). The present work demonstrates the kind of corrections needed in order to obtain hydrostatic from non-hydrostatic static compression results on polycrystals above their yield point. The correction was not applied to the data establishing the secondary ruby scale [13] because the corrections in dynamic and static experiments were thought to cancel. The compression curves shown here were not observed in that work because the pressure steps taken were rather large and because the yield strengths of the directly compressed foils were exceeded below the mini-

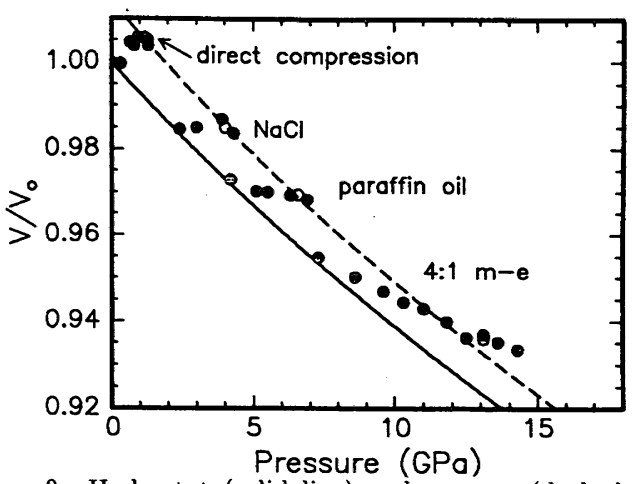

Fig. 3. Hydrostat (solid line) and a curve (dashed line) linking the yield point of $\mathrm{Cu}_{3} \mathrm{Au}$ (open circles) determined in various pressure media. The latter curve was fitted with the equation of state of undeformed $\mathrm{Cu}_{3} \mathrm{Au}$ determined in 4:1 methanol-ethanol to $7 \mathrm{GPa}$ using a constant offset of $1.9 \mathrm{GPa}$ to the hydrostat. The solid circles are the scaled average volumes of $\mathrm{Cu}_{3} \mathrm{Au}$ in different pressure media between the hydrostat and the curve linking the respective yield point. Plastic deformation in liquid media is here caused by the glass transitions.

mum pressure applied (around $7 \mathrm{GPa}$ ). The present results illustrate the problems to be expected in experiments on polycrystals (especially soft reactive metals like the alkali and alkaline earth metals and the lanthanides) and shows that the combination of a DAC with spectroscopic techniques is a powerful method for the non-destructive in-situ investigation of plastic deformation at low rates of strain.

\section{References}

[1] R.A. Forman, G.J. Piermarini, J.D. Barnett and S. Block, Science, 176, 284 (1972)

[2] D. Hull and D.J. Bacon, Introduction to Dislocations, Pergamon Press, Oxford, 1984

[3] J.-P. Poirier, Creep of Crystals, Cambridge Univ. Press, Cambridge, 1985

[4] M. Yamaguchi and Y. Umakoshi, Progress in Materials Science, 34, 149 (1990)

[5] A.K. Singh, J. Appl. Physics, 73, 4278 (1993)

[6] B. E. Warren, X-ray diffraction, Dover Reprint (1990)

[7] J.W. Otto, Nucl. Instrum. Methods, A384, 552 (1997)

[8] J.W. Otto, J. Appl. Cryst., (1997) (accepted)

[9] A. Seeger and P. Haasen, Philos. Mag., 3, 470 (1958)

[10] J.W. Otto, J.K. Vassiliou and G. Frommeyer, J. Synchrotron Rad., 4, 155 (1997)

[11] T. Le Bihan, S. Heathman, S. Darracq, C. Abraham, J.-M. Winand and U. Benedict, High Temperatures-High Pressures, 27/28, 157 (1996)

[12] O.E. Jones and R.A. Graham, NBS Spec. Publ. No. 326, Washington, 1971

[13] H.K. Mao, P.M. Bell, J.W. Shaner and D.J. Steinberg, J. Appl. Phys., 49, 3276 (1978) 